\title{
Heartbeat: Diabetes and heart failure
}

In this issue of Heart, Skrtic and colleagues ${ }^{1}$ evaluated data from over 94 thousand primary care patients with type 2 diabetes (T2D) in the Clinical Practice Research Data Link to study the relationship between incident heart failure (HF) and hemoglobin A1c (HbA1c) levels at the initial diagnosis (baseline), most recent visit (updated latest) and an updated mean level. As shown in figure 1, elevations in all $3 \mathrm{HbA} 1 \mathrm{c}$ levels were associated with a higher hazard ratio for HF, with the strongest association seen for the updated mean value. The authors suggest that 'hyperglycaemia continues to be a strong risk factor of $\mathrm{HF}$ in persons with T2D, and the higher risk estimates for the updated mean HbA1c indicate that there is clinically significant benefit on reducing $\mathrm{HF}$ outcomes by implementing glycaemic control.' They also note that the risk of HF may be underestimated by the baseline or latest HbA1c level.

In the accompanying editorial, ${ }^{2}$ Rahimi reviews the evidence for an association between glycemic control and HF and then discusses the importance of measurement error and time-varying fluctuations in single measurements of HBA1c. Considering the current data in the context of previous studies, his summary comment is that 'Taken together, it seems appropriate to conclude that among patients with type 2 diabetes each 1 percentage point higher 'usual' (ie, long-term average) HbA1c is associated with a $15 \%$ higher risk of HF.' However, he cautions that 'the question of treatment effects and whether the associations between $\mathrm{HbA} 1 \mathrm{c}$ and risk of heart failure are causal are still a matter of debate' and lists several possible non-casual mechanisms for this association. Thus, 'the conclusion from Skrtic et al to call for tighter control of blood glucose in patients with diabetes as a way of reducing the risk of heart failure seems premature.'

Adults with repaired tetralogy of Fallot (rTOF) are at increased risk of arrhythmias and sudden death but the mechanism of this association is unclear. Benoist and colleagues $^{3}$ used an animal model of rTOF

Division of Cardiology, University of Washington, Seattle, USA

Correspondence to Professor Catherine M Otto, Division of Cardiology, University of Washington, Seattle, WA 98195, USA; cmotto@u.washington.edu

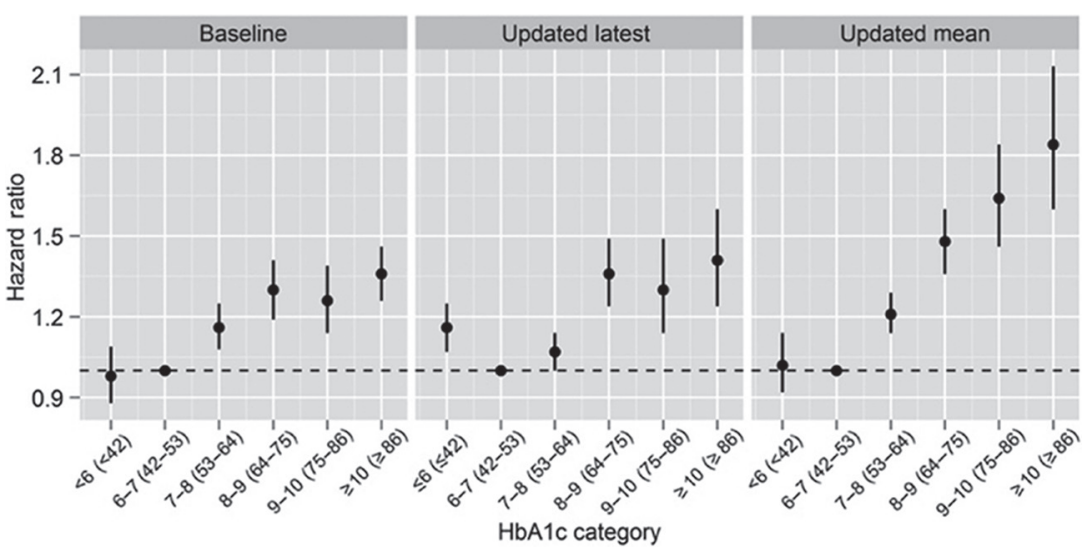

Figure 1 Estimated $\mathrm{HRs}$ with $95 \% \mathrm{Cl}$ for each of the three $\mathrm{HbA} 1 \mathrm{c}$ variables, across categories of $\mathrm{HbA} 1 \mathrm{c}$ level for heart failure events. Reference category is $6 \%-<7 \%(42-53 \mathrm{mmol} / \mathrm{mol})$. The dashed line indicates $H R=1$.

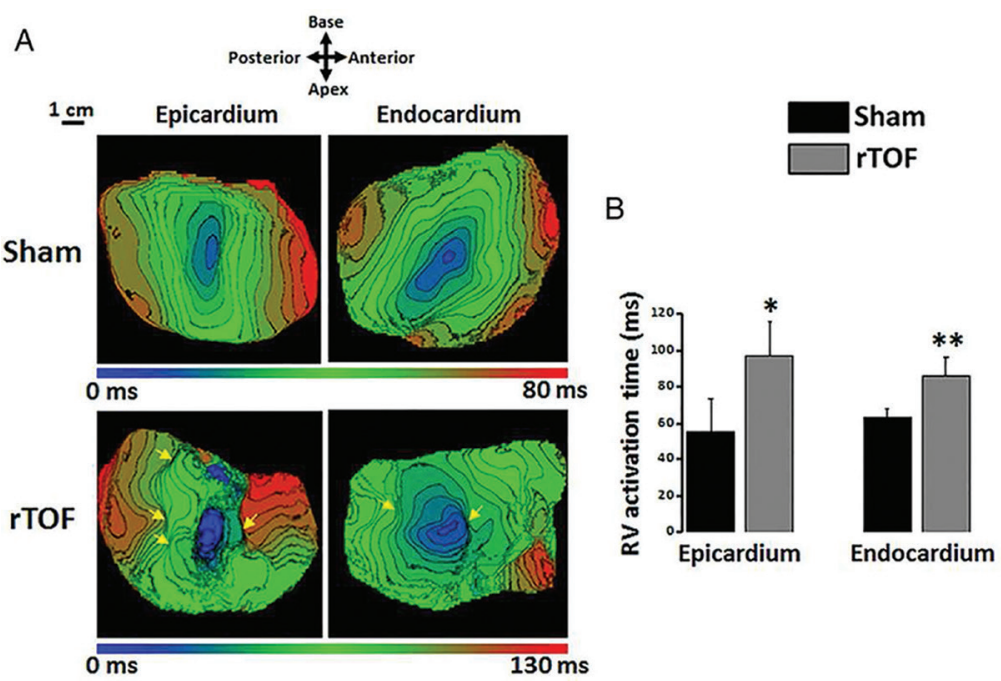

C

D
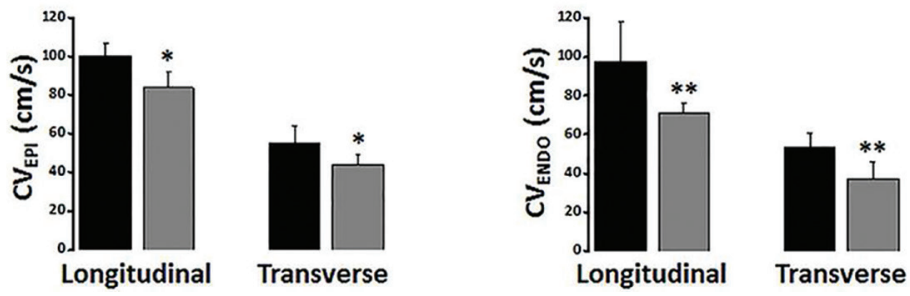

Figure 2 RV activation in Sham and rTOF preparations. (A) Representative activation maps from a Sham and a rTOF RV with 5 ms-spaced isochrones. Multiple areas of slowed conduction were observed (yellow arrows) throughout rTOF RVs. (B) Epicardial and endocardial activation times were longer in rTOF than in Sham RVs. Epicardial (C) and endocardial (D) CVs were reduced in the longitudinal and transverse directions of propagation in rTOF compared with Sham preparations. Data are means $\pm S D$. ${ }^{* *} p<0.01,{ }^{*} p<0.05$, Sham $N=4, r T O F N=5-6$. CV, conduction velocity; ENDO, endocardial; EPI, epicardial; rTOF, repaired tetralogy of Fallot; RV, right ventricle. 
to examine right ventricular (RV) remodeling, conduction velocities, and action potential duration, as well as protein and gene expression (figure 2). Their data suggest a that RV anatomic and electrical remodeling results in a proarrhythmic substrate that likely facilitates re-entry and contributes to the risk of sudden cardiac death.

In an editorial, Seslar and Robinson ${ }^{4}$ discuss the challenges of using animal models to study complex conditions such as rTOF, which has a wide range of disease severity and a variety of approaches to surgical correction. Despite these concerns, they conclude that 'The development of animal models allows elucidation of the genetic and biochemical underpinnings of the disease process, as highlighted in the present study, and the investigation of novel therapies such as nanotechnology and stem-cell therapy. Perhaps most importantly, it shows the value of bringing together a multidisciplinary team to address these complex issues.'

Adults with congenital heart disease often had diagnostic or procedural cardiac catheterisations in childhood with attendant radiation exposure. Harbron and colleagues $^{5}$ estimated lifetime attributable risk (LAR) related to radiation exposure during childhood using radiation doses measured at 2749 procedures performed at 5 UK hospitals and models developed by the Biological Effects of Ionising Radiation committee. This model suggests that the greatest risks associated with cardiac catheterisation are for lung cancer in both sexes and breast cancer in females. Overall LAR is estimated to be 1 in 2000 $(0.05 \%)$ for males and 1 in $500(0.2 \%)$ for women. However, there is consideration variability in LAR depending on age at exposure, type of procedure and expected longevity (figure 3 ). LAR ranges from $<1$ in 2000 for trans-catheter atrial septal defect closure to 1 in 150 for transcatheter valve replacement. Of course, lifetime risk of cancer is lower for patients with more severe congenital heart disease because life expectancy is significantly reduced due to the cardiac condition.

Beausejour-Ladouceur and Lawler comment $^{6}$ that the concern about exposure to low dose radiation (LDIR) in
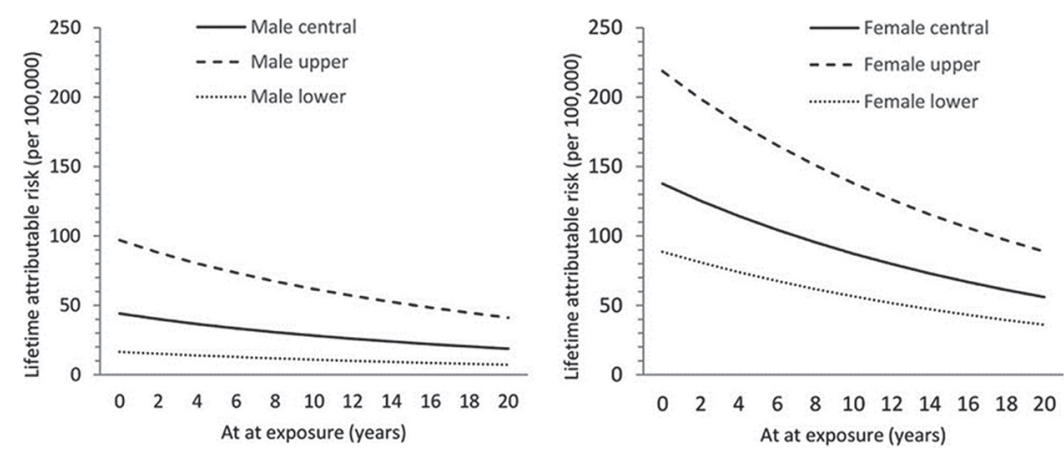

Figure 3 Lifetime attributable risk for all cancers combined as a function of age-at-exposure. Upper and lower bounds are based on $95 \% \mathrm{Cls}$ of elevated relative risk (ERR) and elevated absolute risk (EAR) risk model coefficients. Risks based on average dose for all procedures combined.

childhood stems from several considerations including epidemiologic data for medical and nonmedical radiation exposure, the concern for greater mutagenesis risk in immature organs early in life, and the increased longevity of patients with congenital heart disease. However, despite these concerns 'it is very likely that in most cases, the benefits will clearly outweigh the potential harms associated with these procedures. Clinicians and patients should be wary of foregoing procedures with known benefit for their theoretical risks. By providing specific estimates, the investigators allow readers to make their own judgements about these risks in the context of the potential benefits.' In addition, 'knowing that breast and lung cancers appear to be the greatest contributors to cardiac catheterisation LDIR-related risks, important practices such as close collimation in the lateral projection to exclude breast tissue from the primary radiation field and use of lung 'shuttering' technique can appreciably reduce the radiation doses to these organs and perhaps mitigate some of these risks.'

The Education in Heart article in this issue $^{7}$ reviews clinical aspects of mitochondrial disease including cardiac involvement and current management strategies. Mitochondrial diseases have matrilinear inheritance, result in systemic signs and symptoms and frequently result in metabolic abnormities. Cardiac involvement typically results in heart failure due to a hypertrophic or dilated cardiomyopathy phenotype. Arrhythmias requiring pacemaker implantation or catheter ablation also are common.

In the Image Challenge in this issue, Sims and colleagues ${ }^{8}$ show a classic (but unusual) echo-Doppler finding with several illustrative online videos. This is a case you will not want to miss!

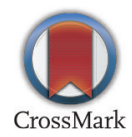

To cite Otto CM. Heart 2017;103:327-328.

Heart 2017;103:327-328.

doi:10.1136/heartjnl-2017-311280

\section{REFERENCES}

1 Skrtic S, Cabrera C, Olsson M, et al. Contemporary risk estimates of three $\mathrm{HbA} 1 \mathrm{c}$ variables in relation to heart failure following diagnosis of type 2 diabetes. Heart 2017;103:355-60.

2 Rahimi K. The long and unfinished journey of hyperglycaemia and heart failure research. Heart 2017;103:331-32.

3 Benoist D, Dubes V, Roubertie F, et al. Proarrhythmic remodelling of the right ventricle in a porcine model of repaired tetralogy of fallot. Heart 2017;103:347-54.

4 Seslar S, Robinson M. Understanding sudden death risk in tetralogy of fallot: from bedside to bench. Heart 2017;103:333-34

5 Harbron RW, Chapple CL, O'Sullivan JJ, et al. Survival adjusted Cancer risks attributable to radiation exposure from cardiac catheterisations in children. Heart 2017;103:341-46.

6 Beauséjour Ladouceur V, Lawler PR. Estimating Cancer risk from invasive cardiac procedures in children. Heart 2017;103:329-30.

7 Limongelli G, Masarone D, Pacileo G. Mitochondrial disease and the heart. Heart 2017:103:390-98

8 Sims JR, Enriquez-Sarano M, Michelena HI. Postoperative dyspnoea. Heart 2017;103:367. 